\title{
SOME EFFECTS OF THE CONSTANT CURRENT UPON TUBERCLE BACILLI IN FLUIDS.
}

\author{
BY CHARLES RUSS, M.B., \\ Assistant Pathologist, Laboratories of Pathology and Public Health.
}

THE effect of electric currents upon bacteria has been studied by various observers, and receives brief notice in several textbooks under consideration of forces not in use in routine laboratory work, such as the effects of light, of great cold, of ultra-spectral rays, and so on. The earlier experiments with currents traversing fluid bacterial suspensions or cultures had a lethal object, doubtless with the object. if electricity proved a potent germicidal agent, of electrolyzing watersupplies and removing the possibility of water-borne disease at the source, or at least the service centre. Water, of course, is practically a non-conductor, and since the addition of salts is necessary for conduction of current, no such germicidal effect, if it were facile in application, would be available, since chemically treated water is useless for a town supply.

In my experiments, which are so far quite embryonic, the effect of currents was observed upon bacteria suspended in fluids. Here, again, for conduction of electricity, chemical salts or electrolytes are necessary, and when these are present-salts of sodium, for example -one observes a movement of the organisms towards one of the electrodes, usually the anode. The common pathogenic species were swept from surface agar growths into distilled water, making strong emulsions, and a few drops of the emulsion added to the common salts of the alkalies arranged in a series of $\mathbf{U}$-tubes furnished with a platinum-foil electrode in each limb. The type of effect was as follows: After a small constant current of 2 or 3 milliampères has traversed the tube for a short time, the bacteria-clouded fluid will be noticed to become denser under the anode if the colon bacillus be used, and as the action continues the aggregation grows until about three-quarters of the tube is bacteria-free, and the organisms are densely massed under the platinum-foil electrode forming the anode.

If at this juncture the current be cut off and the tube and apparatus is simply left alone, the column of aggregated organisms will, so to speak, contract and effect a closer massing under the anode than occurs during continued electrolysis. This effect occurs with living or dead organisms, and apparently the electrode sought by the bacterium depends upon the nature and composition of the dissolved electrolyte for one and the same species of organism. It also appears 
that certain bacteria refuse to move to either electrode in certain electrolytes, and yet migrate to the anode in one and the kathode in another electrolyte.

The behaviour of the tubercle bacillus was especially, but by no means fully, studied in my experiments with a view to utilization of this movement. It appears to be a comparatively inactive organism, and in view of the probable nature of the movement being a chemical affinity between the ions or charged atoms of the chemical used and the bacillus, this inactivity is not unexpected, considering its cultural inertia and comparative indifference to the ordinary staining reagents.

An affinity was observed, however, between the ammonium salts and the substituted ammonias or amines, and by using ethylamine in acid solution, the tubercle bacillus was driven to the kathode, and recognized by stained films prepared from the fluid at the negative pole, while no bacilli could be seen in a similar film from the anode limb. By substituting a glass tube in which the kathode was concealed, tubercle bacilli were drawn from known tuberculous urine into the glass tube, seeking the kathode, and the bacilli recognized in stained films prepared from the fluid inside the glass tube (the tuberculous urine having been previously mixed with the specific electrolyte). While using the chemical affinity indicated above as a working theory of the cause of the effect, it must be remembered that the electric charge upon the moving ions is also a factor in producing the movement. ${ }^{1}$ The uses to which this force may be applied are:

I. From a laboratory standpoint. If this movement be an expression of a chemical affinity for the atoms of the electrolyte (which are known to move to each electrode), then it should be possible to find a series of chemicals in which different bacteria will show differences of behaviour (by movement or stasis when electrified), and such differences would be evidence of identity.

2. The current is apparently a delicate detective agent in cases in which a few bacteria - viz., tubercle bacilli-are present in a fluid, and the application for extracting bovine tubercle bacilli from tuberculous milk is at present engaging attention, since, if successful, they should be found in films and obviate the long delay before inoculation results are available.

1 The form of apparatus employed in these researches I have illustrated elsewhere. See "The Electrical Reactions of Bacteria applied to the Detection of Tubercle Bacilli in Urine by Means of a Current," Lancet, July 3, I909. The apparatus was also exhibited in the Museum of the British Medical Association, I9Io. 